\title{
Caracterização da cobertura do pré-natal no Estado do Maranhão, Brasil
}

\author{
Coverage characterization of pre-natal in Maranhão State, Brazil \\ Caracterización de la cobertura del pre-natal en lo Estado de Maranhão, Brasil
}
Geny Rose Cardoso Costa', Maria Bethânia da Costa Chein', Mônica Elinor Alves Gama', Leidyane Silva Caldas Coelho', Andreia Susana Vieira da Costa', Carlos Leonardo Figueiredo Cunha', Luciane Maria Oliveira Brito'

'Universidade Federal do Maranhão. Programa de Pós-graduação em Saúde Materno-Infantil. São Luís, MA

Submissão: 1 1/09/2009

Aprovação: 17/10/2010

\section{RESUMO}

O objetivo do estudo foi caracterizar a cobertura do pré-natal no Estado do Maranhão. Um estudo de base populacional em 30 municípios do Maranhão, com 2.075 mulheres em idade fértil, com gravidez prévia, de julho de 2008 a janeiro de 2009. Os resultados mostraram que as unidades de saúde da família foram responsáveis por 45,9\% dos atendimentos de gestantes sendo que 46,8\% relataram a realização de seis consultas ou mais de pré-natal durante a última gravidez e $64,6 \%$ iniciaram o pré-natal ainda no $1^{\circ}$ trimestre. A cobertura do pré-natal foi de $85,6 \%$, no entanto, ao se considerar a cobertura de pré-natal adeQuado, este foi de $43,4 \%$, de acordo com o preconizado pelo Ministério da Saúde (Brasil). Apesar de uma cobertura de pré-natal acima de $80 \%$, menos da metade é considerada adeQuada, demonstrando um descompasso na atenção primária com Qualidade.

Descritores: Assistência pré-natal; Saúde da mulher; Serviço de Saúde.

\section{ABSTRACT}

The purpose of the study was to characterize the coverage of prenatal care in the State of Maranhão. A population-based study, descriptive in 30 municipalities of the State of Maranhão, with 2075 women of childbearing age, with previous pregnancy, from July 2008 to lanuray 2009. The results demonstrated that the units of family health accounted for $45.9 \%$ of the care of pregnant women and that $46.8 \%$ reported carrying out consultations six or more prenatal care during last pregnancy and $64.6 \%$ started prenatal in the first three months of pregnancy. The coverage of prenatal care, without regard to adequacy, was $85.6 \%$, however, when considering the coverage of adequate prenatal as established by Brazilian Health Ministry was $43.4 \%$. Although coverage of prenatal above $80 \%$, less than half is considered adeeuate, showing a gap in primary care Quality.

Keywords: Prenatal care; Women's Health; Health service.

\section{RESUMEN}

El objetivo de este estudio fue caracterizar la cobertura de atención prenatal en el Estado de Maranhão. Un estudio de base poblacional en 30 municipios del Estado de Maranhão, con 2.075 mujeres en edad fértil, con el embarazo anterior, entre julio de 2008 enero de 2009. Los resultados mostraron Que las unidade de salud de la familia fueron responsables de un $45,9 \%$ de la atención de la mujer embarazada y Que el 46,8\% indicaron Que hacían seis o más visitas a la atención prenatal durante su último embarazo y el 64,6\% comenzó la atención prenatal sigue siendo en el ler trimestre. La cobertura de atención prenatal fue de 85,6\%, sin embargo, cuando se considera la cobertura de atención prenatal adecuada, esto fue un $43,4 \%$, de acuerdo con las recomendaciones del Ministerio de Salud (Brasil). Aunque la cobertura de atención prenatal por encima del $80 \%$, menos de la mitad se considera satisfactoria, con una brecha en la calidad de la atención primaria.

Descritores: Atención prenatal; Salud de la Mujer; Servicio de salud.

AUTOR CORRESPONDENTE Programa de Pós-Graduação em Saúde Materno-Infantil da Universidade Federal do Maranhão, Praça Gonçalves Dias, 21, Centro. CEP 65020-240. São Luís, MA. E-mail: ppgsmin@ufma.br 


\section{INTRODUÇÃO}

O Programa de Saúde da Família (PSF) desde sua implantação em 1994 obteve um aumento expressivo no seu crescimento. Nos primeiros Quatro anos de implantação, o PSF assistia a 3,51\% da população brasileira, em abril de 2008, esta cobertura representou $56,8 \%$, ou seja, cerca de 107 milhões de brasileiros ${ }^{(1)}$.

Essa assistência realizada pelo PSF melhorou em todo o país. No Maranhão em 1998, ano de sua implantação, existiam 10 Equipes de Saúde da Família, com uma cobertura de 0,7\%, já ao final de 2007, cobria 75,5\% da população, com 1.619 equipes $^{(2)}$.

Esses dados expressam a melhoria da oferta de serviços prestada pelo PSF, o Que colaborou diretamente no aumento da utilização destes serviços pela população, e conseQüentemente uma melhor cobertura na assistência pré-natal.

Cabe ressaltar Que o pré-natal deve ser organizado para atender às reais necessidades da população de gestantes por meio da utilização de conhecimentos técnico-científicos e recursos adequados e disponíveis para cada caso. Reforça-se, ainda, que as ações de saúde precisam estar voltadas para cobertura de toda a população alvo da área de abrangência da unidade de saúde, assegurando a continuidade no atendimento, o acompanhamento e a avaliação dessas ações sobre a saúde materna-perinatal ${ }^{(3)}$.

A gravidez é reconhecidamente um dos determinantes do estado de saúde da mulher, sendo em algumas situações o único momento de contato Que a mulher em idade reprodutiva terá com os serviços de saúde, tratando-se de uma grande oportunidade para uma assistência direcionada à promoção da saúde da mulher, orientação e rastreamento de enfermidades.

Neste sentido, visando garantir, entre outras, a identificação precoce de todas as gestantes da comunidade e o pronto início do acompanhamento no primeiro trimestre da gravidez, bem como a operacionalização do sistema de referência e contra-referência, foram estabelecidas condições para uma assistência pré-natal efetiva objetivando garantir a continuidade da assistência em todos os níveis de complexidade do sistema de saúde ${ }^{(3)}$.

Não obstante a essas estratégias e conceitos, o início da assistência prénatal em várias regiões do Brasil ainda é tardio ${ }^{(4-5)}$, e o número de consultas é deficiente, verificando-se grande desigualdade entre as regiões do país, considerando-se o número mínimo de sete consultas, observa-se a seguinte distribuição: Norte 30, $18 \%$, Nordeste $39,33 \%$, Sudeste $68,69 \%$, Sul $70,79 \%$, Centro Oeste $60,38 \%$, e a média no Brasil de $55,38 \%{ }^{(6)}$.

O pré-natal com Qualidade destaca-se como sendo o primeiro alvo a ser atingido Quando se busca reduzir as taxas de morbimortalidade materna e perinatal e, para tanto, a viabilização dos programas exige atuação profissional competente e atualizada de modo contínuo.

O estudo objetiva identificar a cobertura da assistência pré-natal no Estado do Maranhão com vistas a acrescentar informações Que possam direcionar à adoção de estratégias diferenciadas, na cobertura e Qualidade da assistência.

\section{MÉTODO}

Realizou-se estudo de base populacional, descritivo, com abordagem Quantitativa em 30 municípios do Estado do Maranhão no período de julho de 2008 a janeiro de 2009 . Foram entrevistadas 3.076 mulheres e selecionadas $2.075(67,45 \%)$ com os seguintes critérios de inclusão: idade reprodutiva entre 10 e 49 anos e relato de gravidez prévia. As informações coletadas sobre assistência prénatal relacionavam-se com a última gravidez ocorrida.

O percentual de perdas e recusas não ultrapassou 5\%, estando abaixo do referencial de $10 \%$ a mais, computado para o tamanho amostral.

Utilizou-se um processo de amostragem aleatória sistemática de conglomerados, em estágios múltiplos, com três etapas. $\mathrm{Na}$ primeira etapa foram sorteados os municípios do Estado do Maranhão, sendo elaborada uma listagem acumulada de municípios e suas respectivas populações, estimadas para o ano de 2006, de acordo com o último Censo Demográfico do Instituto Brasileiro de Geografia e Estatística (IBGE), disponibilizado pela instituição.

No segundo estágio foram sorteados os setores censitários dentro de cada município, a partir de uma listagem dos setores fornecida pelo IBGE. Em cada conglomerado foram sorteados sete setores para visitação e mais dois setores reservas, a serem utilizados em casos excepcionais, Quando da impossibilidade de acesso ao setor sorteado (limitação climática ou geográfica).

No terceiro estágio foi sorteado o ponto inicial dentro de cada setor, a partir do Qual certo número de domicílios foi visitado. De acordo com o mapa do setor, os Quarteirões neles existentes foram numerados e realizados o sorteio do Quarteirão inicial para visita. As Quatro esquinas do Quarteirão sorteado foram identificadas com letras A, B, C e D, efetuando-se o sorteio da letra e a esquina sorteada foi o ponto inicial. A partir da casa da esQuina o Quarteirão
Quadro 1. Categorias de adeQuação das mulheres em relação à assistência pré-natal, baseado no calendário mínimo preconizado pelo Ministério da Saúde. 
foi percorrido em sentido horário e as casas foram visitadas até encontrar 16 mulheres entre 10 a 49 anos com relato de gravidez prévia, em cada conglomerado. Em caso de conglomerados com traçado irregular, de 3 a 5 letras foram colocadas no mapa, a partir de pontos de referência identificados após uma visita ao local, sendo realizado sorteio do ponto inicial.

Para a classificação de cobertura do pré-natal foram utilizadas as categorias de adeQuação: casos ignorados, pré-natal ausente, inadequado, intermediário e adequado, a fim de comparar com trabalho, com metodologia similar, realizada em década anterior ${ }^{(7)}$ (Quadro 1).

Utilizou-se um Questionário com variáveis Que contemplavam a assistência à mulher no tocante ao uso de serviço de assistência pré-natal no âmbito público e privado e características do prénatal, considerando-se o número e o mês de início das consultas em relação aos nove meses gestacionais.

Para digitação e processamento de dados utilizou-se o programa Epi-Info 2.002 do CDC, de Atlanta, EUA. Os dados foram analisados com o programa SPSS for Windows 10.0. Esses dados foram submetidos a técnicas de estatística descritiva não inferencial, permitindo, assim, uma análise exploratória através de gráficos de freqüência e de todas as variáveis investigadas na pesquisa. Em todos os testes o nível de significância foi de $5 \%$ ( $p<0,05)$.

A pesquisa foi aprovada pelo Comitê de Ética em Pesquisa do Hospital Universitário da Universidade Federal do Maranhão com o parecer no 307/2006.

\section{RESULTADOS E DISCUSSÃO}

Observou-se Que a maioria das mulheres encontrava-se entre 20 a 29 anos de idade $(38,0 \%)$, residia na zona urbana $(53,5 \%)$, era alfabetizada $(81,5 \%)$, com um a Quatro anos de escolaridade (3 1,0\%), apresentava união estável $(42,2 \%)$ ou era legalmente casada $(36,8 \%)$, não apresentava atividade laboral extra-residencial remunerada $(52,0 \%)$ e tinha como renda familiar menos de um salário mínimo (47,0\%).

O Sistema ùnico de Saúde (SUS)foi responsável por 78,4\% (1.353) dos atendimentos de pré-natal, sendo $76,2 \%$ no setor exclusivamente público e 2,2\% no setor conveniado ou filantrópico, já 4,5\% (80) e $0,7 \%$ (12) das consultas foram, respectivamente, particulares e utilizando os planos de saúde.

As Unidades Básicas de Saúde (UBS), atendidas pelo Programa de Saúde da Família (PSF), foram responsáveis pela realização de $45,9 \%$ (62 I) da assistência pré-natal, dentre o universo de 1.353 daquelas que realizaram pré-natal no SUS (1.353 mulheres).

Nas regiões brasileiras, observam-se semelhanças no uso dos serviços, sendo a maioria na rede pública de saúde com a procura de consultas preventivas, enQuanto Que os não usuários utilizam pronto-socorro e hospitais - ambos independentes do sexo feminino ou masculino ${ }^{(8)}$. É necessário enfatizar Que o uso resolutivo dos serviços de saúde depende da associação de fatores individuais e internos destes serviços Que são refletidos na Qualidade da assistência ${ }^{(9)}$.

No período de 1992 a 1999 o nº de estabelecimentos públicos cresceu $24,9 \%$, enquanto Que os privados apresentaram uma redução de $2,8 \%$. Ressalte-se Que este aumento ocorreu predominantemente na rede municipal, onde de 69,0\% em 1992 passou para $92 \%$ em $1999^{(10-1)}$.

O uso regular de serviços de saúde cresceu, na população brasileira entre 1998 e 2003 , de 13\% para 14,6 \%, e esse aumento foi devido a ofertas de serviços públicos de saúde pelo SUS e não especificamente ao sistema de saúde suplementar, Que se manteve inalterado neste período ${ }^{(10-11)}$.

O pré-natal apresentou uma cobertura de 85,6\% (1.776) em relação ao universo de 2.075 mulheres selecionadas, observou-se Que $64,6 \%$ (1.147) iniciaram o pré-natal antes do término do primeiro trimestre gestacional, no entanto, menos da metade, $43,4 \%$ (770), o fizeram de forma adequada, ou seja, iniciaram o pré-natal no primeiro trimestre com no mínimo seis consultas para uma gestação a termo.

Quanto ao número de consultas, $46,8 \%$ das mulheres realizou seis ou mais consultas pré-natais, resultado semelhante ao da pesquisa realizada há 10 anos, com 43,9\% de adequação do prénatal (na ocasião o MS preconizava cinco ou mais consultas) ${ }^{(3,7)}$.

Apesar de um maior contingente de mulheres esteja realizando o pré-natal, o seu início precoce ainda continua um desafio no modelo proposto pelo Ministério da Saúde, onde todas as mulheres iniciam a primeiro consulta até a $12^{\text {a }}$ semana de gravidez e que tenha durante o pré-natal pelo menos seis consultas ${ }^{(3)}$.

A expansão de cobertura cada vez maior alcançada pelo PSF, o Qualifica como fundamental para o desenvolvimento da atenção básica em todo o país; seu impacto nos indicadores de saúde é marcante. Em relação à assistência pré-natal seu impacto é observado diretamente pela redução da mortalidade materna e infantil ${ }^{(3,12)}$.

O número de consultas pré-natais muitas das vezes pode refletir o início do pré-natal, pois Quanto mais cedo for, a Qualidade será melhor e a adesão ao pré-natal se fará refletir em um número maior de consultas $^{(12-13)}$.

AQuelas Que não realizaram nenhuma consulta representaram $14 \%$ das entrevistadas, pesQuisa realizada há uma década observou 9,3\% entre as parturientes em idade fértil de hospitais de referência no Estado ${ }^{(14)}$. Esses dados ainda podem ser subregistrados, pois a pesquisa não contemplou aquelas mulheres que haviam tido o seu parto fora do hospital.

Os resultados sugerem Que a implantação do Programa de Humanização do Pré-Natal (PHPN) de 2000 no Maranhão, juntamente com o PSF e Agentes Comunitários de Saúde, trouxe como consequência positiva o aumento de mulheres que tiveram agendamento prévio na sua marcação de consulta de pré-natal ${ }^{(15)}$.

O percentual de mães Que iniciaram precocemente o pré-natal elevou-se de 60,1\% em 1991, para 69,8\% em 1997(7), porém nesta pesquisa observou-se um decréscimo para $59,8 \%$, resultado não esperado, já Que houve um aumento considerável da cobertura oferecido pela estratégia saúde da família no Maranhão, Que atingiu $85,6 \%$. Entretanto, essa captação deficiente no primeiro trimestre gestacional ao serviço público de saúde é também uma preocupação em outras cidades.

Na cidade de Juiz de Fora em Minas Gerais, o SUS captou para início de pré-natal no primeiro trimestre somente $29,7 \%$ das gestantes $^{(16)}$, já nas cidades de Caxias do Sull ${ }^{(17)}$ e Pelotas $^{(18)}$, ambas no Rio Grande Sul, observaram-se $34,7 \%$ e cerca de $46 \%$, respectivamente. Considerando ser este Estado da federação, o Quarto Que apresenta o melhor Índice de Desenvolvimento Humano 
$(\text { IDH })^{(19)}$ seria plausível Que nestas cidades houvesse uma maior freqüência da assistência pré-natal no primeiro trimestre, no entanto, ao comparar-se com o Maranhão, o segundo pior Estado em IDH ${ }^{(19)}$, a cobertura foi de $64,6 \%$.

A captação precoce das grávidas no pré-natal é um fator de extrema importância para a saúde das mulheres e de recém-nascidos, pois possibilita a identificação antecipada da gestação de risco, bem como as intervenções necessárias, pois o início tardio e a realização de menor $\mathrm{n}^{\circ}$ de consultas podem comprometer um dos principais trabalhos desenvolvidos durante o pré-natal Que a promoção da saúde ${ }^{(3,12,21)}$.

Menos de $50 \%$ das mulheres utilizaram o serviço de pré-natal adequadamente; estes resultados expressam Que, apesar do Que preconiza a Portaria Ministerial, no569 \GM de 0 I \06\2000, Quanto ao início da assistência pré-natal até o final do primeiro trimestre gestacional com no mínimo seis consultas por período gestacional $^{(3,12,21)}$, ainda falta muita sensibilização da população acerca da importância de um pré-natal adequado, remete também a um Questionamento sobre a atuação dos gestores municipais de saúde, Que são os diretamente responsáveis, após a municipalização do SUS, por esta prestação de serviços ${ }^{(21)}$.

Cumpre destacar Que o acompanhamento pré-natal com a realização de seis ou mais consultas garante uma evolução saudável da gestação aliada à precocidade de detecção de eventuais fatores de riscos à mãe e ao bebê, possuindo a mesma importância o envolvimento da gestante a sua família no intuito de oportunizar a vivência desse momento e prepará-los para o parto e puerpério $^{(3,12,21)}$, e com a atuação dos profissionais do PSF vem permitindo Que se estabeleça vínculos com as gestantes Que melhoram a adesão das mesmas ao Programa de Assistência PréNatal (PAPN) ${ }^{(22)}$.

No Maranhão, a ausência da realização do pré-natal vem diminuindo, passando de $41,0 \%$ em 1991 ${ }^{(7)}, 28,7 \%$ em 1997(7) $\mathrm{e}$ $14,4 \%$ observada nesta pesquisa. Esses dados associam-se à expansão do acesso às consultas de pré-natal, sugerindo Que houve uma coordenação na captação das gestantes, promovido e acelerado pela estratégia saúde da família, com equipes de referência em obstetrícia, onde a enfermagem e os agentes comunitários considerados esteios, alocadas de forma descentralizada nos pólos e nas unidades de atenção especializada à mulher determinam o aumento da cobertura de pré-natal.

\section{CONSIDERAÇÕES FINAIS}

Sendo a cobertura pré-natal um indicador de Qualidade da atenção básica em saúde, esta pesQuisa revela Que nos últimos 10 anos houve um aumento significativo. Entretanto, destacam-se desafios necessários ao aperfeiçoamento da atenção às mulheres gestantes nas suas distintas demandas.

Os resultados desta pesquisa, Que abrangeram todo o território maranhense, não podem evidentemente ser extrapolados para toda a população brasileira, haja vista as peculiaridades nos aspectos políticos, econômicos, geográficos e sociais deste Estado. Seus achados conferem a importância Que tem o tema, e apontam claramente para a necessidade de incorporar a responsabilidade nas Políticas de Saúde, Que dêem conta das especificidades da mulher contribuindo de forma expressiva na assistência ao prénatal no Estado do Maranhão.

\section{REFERÊNCIAS}

1. Ministério da Saúde (BR). Departamento de Atenção Básica. Evolução da Implantação das Equipes Saúde da Família no Brasil - 1998 a Abril/2008. [citado em 2009 Jun 20]. Disponível: http://dab.saude.gov.br/dab/abnumeros.php

2. Marques RM. A atenção Básica e Programa de Saúde da Família (PSF): novos rumos para a política de saúde e seu funcionamento. Ciênc Saúde Coletiva 2003; 8(2): 403-15.

3. Ministério da Saúde (BR). Secretaria de Atenção à Saúde. Departamento de Ações Programáticas Estratégicas. Área Técnica de Saúde da Mulher. Pré-natal e Puerpério: atenção Qualificada e humanizada - manual técnico. Brasília: Ministério da Saúde; 2005.

4. Saliba MSA, Saliba GCA, Isper GAJ, Guimarães ZL, Donha YS, Soares FKM. Sistema de Informação Pré-Natal: análise crítica de registros em um município paulista. Rev Bras Enferm 2010; 63(3): 385-90.

5. Lana FCFL, Silva A. Avaliação da prevenção da transmissão vertical do HIV em Belo Horizonte, MG, Brasil. Rev Bras Enferm 2010; 63(4): 587-94

6. Ministério da Saúde (BR). Rede Interagencial de Informações para a Saúde - RIPSA. Indicadores e Dados Básicos - Brasil 2008. Cobertura de consultas de pré-natal. [citado emn 2009 Jun 20]. Disponível: http://tabnet.datasus.gov.br/cgi/ deftohtm.exe?idb2008/f06.def

7. Coimbra LC, Mochel EG. Assistência pré e perinatal. In: Tonial
SR, Silva AAM, organizadores. Saúde, Nutrição e Mortalidade Infantil no Maranhão. São Luís: Universidade Federal do Maranhão/ Secretaria de Estado da Saúde/UNICEF; 1997. p. 30-35.

8. Ribeiro MCSA, Barata RB, Almeida MF, Silva ZP. Perfil sociodemográfico e padrão de utilização de serviços de saúde para usuários e não-usuários do SUS - PNAD 2003. Ciênc Saúde Coletiva 2006; 1 I (4): 10 1 I-22.

9. Travassos C, Martins M. Uma revisão sobre os conceitos de acesso e utilização de serviços de saúde. Cad Saúde Pública 2004, 20(sup 2): S190-S98.

10. Noronha JC, Lima LD, Machado CV. O Sistema Único de Saúde (SUS). In: Giovanella L, Escorel S, Lobato LVC, Noronha IC, Carvalho AI. Políticas e sistema de saúde no Brasil. Rio de Janeiro: Fiocruz; 2008. p.435-72.

11. Ministério da Saúde (BR). Cadastro Nacional de Estabelecimentos de Saúde. [citado em 2010 Out 09]. Disponível: http:/http://cnes.datasus.gov.br/Index.asp?home = I

12. Ministério da Saúde (BR). Secretaria de Atenção à Saúde. Departamento de Ações Programáticas Estratégicas. Política nacional de atenção integral à saúde da mulher: princípios e diretrizes. Brasília: Ministério da Saúde; 2007.

13. Ministério da Saúde (BR). Secretaria de Vigilância em Saúde. Departamento de Análise de Situação em Saúde. Saúde Brasil 2006: uma análise da situação de saúde no Brasil. Brasília: Ministério da Saúde; 2006. 
14. Coimbra LC, Silva AAM, Mochel EG, Alves MTSSB, Ribeiro VS, Aragão VME, et al. Fatores associados à inadęuação do uso da assistência pré-natal. Rev Saúde Pública 2003; 37 (4): 456-62.

15. Carvalho V, Araújo TVB. AdeQuação da assistência pré-natal em gestantes atendidas em dois hospitais de referência para gravidez de alto risco do Sistema único de Saúde, na cidade de Recife, Estado de Pernambuco. Rev Bras Saúde Materno Infantil 2007; 7(3): 309-17.

16. Coutinho T, Teixeira MTB, Dain S, Sayd ID, Coutinho LM. AdeQuação do processo de assistência pré-natal entre as usuárias do Sistema Único de Saúde em Juíz de Fora MG. Rev Bras Ginecol Obstet 2003; 25: 717-24.

17. Trevisan MR, De Lorenzi DRS, Araujo NM, Ésber K. Perfil da Assistência Pré Natal entre usuárias do Sistema Único de Saúde em Caxias do Sul. Rev Bras Ginecol Obstet 2002; 119 (1):33-42.

1 8. Silveira DS, Santos IS. AdeQuação do pré-natal e peso ao nascer: uma revisão sistemática. Cad Saúde Pública 2004; 20: I I 60-8.
19. Organização das Nações Unidas. Programa das Nações Unidas para o Desenvolvimento Humano (PNUD). Ranking do IDH dos Estados em 2005. [citado em 2008 Set 15]. Disponível em: http://www.pnud.org.br/pobreza_desigualdade/ reportagens/index.php?id0 I = 3039\&lay $=$ pde .

20. Carvalho VCP, Araújo TVB. Adeeuação da assistência pré-natal em gestantes atendidas em dois hospitais de referência para gravidez de alto risco do Sistema único de Saúde, na cidade de Recife, Estado de Pernambuco. Rev Bras Saúde Matern Infant 2007; 7(3): 309-17.

21. Ministério da Saúde (BR). Secretaria de Atenção à Saúde. Departamento de Ações Programáticas Estratégicas. Relatório de gestão 2003 a 2006: política nacional de atenção integral à saúde da mulher. Brasília: Ministério da Saúde; 2007.

22. Gonçalves R, Urasaki MBM, Merighi MAB, D'Avila CG. Avaliação da efetividade da assistência pré-natal de uma Unidade de Saúde da Família em um município da Grande São Paulo. Rev Bras Enferm 2008; 6I(3): 349-53. 